\title{
A RANGE THEOREM FOR THE RADON TRANSFORM
}

\author{
W. R. MADYCH AND D. C. SOLMON \\ (Communicated by Richard R. Goldberg)
}

\begin{abstract}
Conditions are prescribed for a function $g$ which are sufficient to ensure that it is the Radon transform of a continuous function $f$ on $\mathbf{R}^{n}$ such that $f(x)=O\left(|x|^{-n-k-1}\right)$ as $|x| \rightarrow \infty$. Roughly speaking, these criteria involve smoothness and the classical polynomial consistency conditions up to order $k$ on $g$. In particular, the result implies Helgason's Schwartz theorem for the Radon transform [Acta Math. 113 (1965)].
\end{abstract}

1. Introduction. The Radon transform of an integrable function $f$ on $\mathbf{R}^{n}$ is defined by

$$
R_{\theta} f(t)=\int_{\langle x, \theta\rangle=t} f(x) d x .
$$

Here $\theta$ is a direction, i.e., a point on the unit sphere $S^{n-1}, t$ is a real number and the integral is over the hyperplane orthogonal to $\theta$ and passing a directed distance $t$ from the origin. We address the questions of when a given function $g$ on $S^{n-1} \times \mathbf{R}$ is the Radon transform of a function $f$ and what regularity and decay conditions on $f$ can be deduced from those of $g$. The fundamental result on this question is due to Helgason [3]. His theorem says that an even function $g$ in the Schwartz space $S\left(S^{n-1} \times \mathbf{R}\right)$ is the Radon transform of a function $f$ in the Schwartz space $S\left(\mathbf{R}^{n}\right)$ if and only if

$$
\int_{-\infty}^{\infty} g(\theta, t) t^{j} d t=P_{j}(\theta), \quad j=0,1, \ldots,
$$

is representable as a homogeneous polynomial of degree $j$ in $\theta$. He also showed that under the above hypotheses $f$ has compact support if and only if $g$ does and that the convex hull of the support of $f$ is determined by the support of $g$. The first result is called the Schwartz theorem for the Radon transform and the second the Paley-Wiener theorem for the Radon transform [4]. An $L^{2}$ version of the Paley-Wiener theorem for the Radon transform was obtained by Lax and Phillips [6]. Smith et al. [9] showed that any even function in the Sobolev space $H^{s}\left(S^{n-1} \times \mathbf{R}\right), s=(n-1) / 2$, is in the range of the closure $\bar{R}$ of the Radon transform as an unbounded operator on $L^{2}\left(\mathbf{R}^{n}\right)$, but that $\bar{R}$ is not necessarily defined by an absolutely convergent integral. Recently, Solmon [10] showed that

Received by the editors September 1, 1987.

1980 Mathematics Subject Classification (1985 Revision). Primary 44A15, 26B40.

Key words and phrases. Radon transform, polynomial consistency condition, asymptotic behavior.

The first author was supported by Air Force Office of Scientific Research Grant No. AFOSR86-1045.

The second author was supported by NSF Grant No. DMS- 8602300 and Consiglio Nazionale Delle Ricerche, Firenze, Italia. 
any even $g$ in $S\left(S^{n-1} \times \mathbf{R}\right)$ is the Radon transform of a $C^{\infty}$ function $f$ such that $f(x)=O\left(|x|^{-n}\right)$ as $|x| \rightarrow \infty$, and that $f(x)=O\left(|x|^{-n-k-1}\right)$ if and only if (1.1) holds for $j=0,1, \ldots, k$.

In this paper we show that a sufficiently smooth even function on $S^{n-1} \times \mathbf{R}$ which together with a finite number of derivatives decays sufficiently fast at $\infty$ is the Radon transform of a continuous function $f$ that is $O\left(|x|^{-n}\right)$ as $|x| \rightarrow \infty$, and show how satisfying a finite number of the conditions (1.1) influences the behavior of $f$ at $\infty$. (Of course, precise conditions on the smoothness and decay are given.) In particular, the result given here implies the Schwartz theorem of Helgason and the recent extension by Solmon. Our proof is based on a result on the asymptotic behavior of the Fourier transform due to Madych [7], rather than the Radon inversion formula as in [10]. This approach is independent of dimension, shorter and simpler than that in [10]. Moreover, the theorem given here is more general.

2. Operators and formulas. Let $\theta$ be a point on the unit sphere $S^{n-1}$ in $\mathbf{R}^{n}$, $n \geq 2$, and $t$ be a real number. The Radon transform of an integrable function $f$ on $\mathbf{R}^{n}$ in the direction $\theta$ at the point $t$ is the function

$$
R f(\theta, t)=R_{\theta} f(t)=\int_{\langle x, \theta\rangle=t} f(x) d x
$$

where $\langle$,$\rangle denotes the usual Euclidean inner product on \mathbf{R}^{n}$ and $d x$ represents integration with respect to $n-1$ dimensional Lebesgue measure on the hyperplane $\langle x, \theta\rangle=t$.

The Fourier transform of an integrable function $f$ on $\mathbf{R}^{n}$ is defined by

$$
\hat{f}(\xi)=(2 \pi)^{-n / 2} \int f(x) e^{-i\langle x, \xi\rangle} d x
$$

where the integral is taken over all of $\mathbf{R}^{n}$. Fixing $\theta$ in (2.1) and taking the one dimensional Fourier transform gives the well-known formula

$$
\left(R_{\theta} f\right)^{\wedge}(\tau)=(2 \pi)^{(n-1) / 2} \hat{f}(\tau \theta) .
$$

The operator $\Lambda$ is defined in terms of Fourier transforms by

$$
(\Lambda f)^{\curlyvee}(\xi)=|\xi| \hat{f}(\xi)
$$

so that $\Lambda^{2}=-\Delta$, where $\Delta$ is the Laplacian. We shall also use the CalderónZygmund representation of $\Lambda,[\mathbf{1}]$,

$$
\Lambda=\sum_{j=1}^{n} \mathscr{H}_{j} D_{j}
$$

where $D_{j}$ denotes partial differentiation with respect to the $j$ th coordinate and $\mathscr{K}_{j}$ is the singular integral operator defined by convolution with the kernel $c_{n} x_{j} /|x|^{n+1}, c_{n}$ a constant depending only on $n$; i.e., $\mathscr{H}_{j}$ is the $j$ th Riesz transform.

The dual Radon transform is the formal adjoint of the Radon transform and is defined on functions on $S^{n-1} \times \mathbf{R}$ by

$$
R^{t} g(x)=\int_{S^{n-1}} g(\theta,\langle x, \theta\rangle) d \theta
$$


The Radon transform, dual Radon transform, and Calderon-Zygmund operator are related by the Radon inversion formula

$$
f=2^{-1}(2 \pi)^{1-n} \Lambda^{n-1} R^{t} R f .
$$

REMARK 2.7. If $f \in L^{p}\left(\mathbf{R}^{n}\right), 1<p<n /(n-1)$, then for almost every $\theta, R_{\theta} f(t)$ exists for almost every $t$ and for a.e. $\theta(2.2)$ is valid for a.e. $\tau$. Also (2.6) holds almost everywhere on $\mathbf{R}^{n}$. See $[\mathbf{1 0}]$.

We use the standard multi-index notation. Thus if $\nu$ is a multi-index i.e. an $n$-tuple of nonnegative integers, $x \in \mathbf{R}^{n}$, and $D=\left(D_{1}, \ldots, D_{n}\right)$, then $x^{\nu}=$ $x_{1}^{\nu_{1}} \cdots x_{n}^{\nu_{n}}, D^{\nu}$ is the differential operator $D_{1}^{\nu_{1}} \cdots D_{n}^{\nu_{n}}$, and $|\nu|=\nu_{1}+\cdots+\nu_{n}$. Also, $D_{\theta}^{j}$ denotes a $j$ th order (tangential) differential operator on the sphere $S^{n-1}$ with smooth coefficients and $g^{(j)}(\theta, t)$ denotes the $j$ th order partial derivative of $g(\theta, t)$ with respect to $t$.

A function $f$ on $\mathbf{R}^{n}$ will be said to be $O\left(|x|^{-m}\right)$ as $|x| \rightarrow \infty$ if there exist constants $C, M>0$ such that $|f(x)| \leq C|x|^{-m}$ whenever $|x| \geq M$. The analogous definition is taken for the statements $f(x)=O\left(|x|^{-m}\right)$ as $|x| \rightarrow 0$, and $g(\theta, t)=O\left(|t|^{-m}\right)$ as $|t| \rightarrow \infty$ or 0 .

The proof of the theorem on the range of the Radon transform is based on the following asymptotic result about Fourier transforms which is a straightforward generalization of [7, Proposition 5].

LEMMA. Let $f$ be a function on $\mathbf{R}^{n}$ such that $\hat{f}$ is integrable. Suppose that $\alpha \geq 0, m$ is an integer satisfying $m>n+\alpha$, and that

(i) $|\xi|^{-\alpha} \hat{f}(\xi)$ is in $C^{m}\left(\mathbf{R}^{n} \backslash\{0\}\right)$, and

(ii) $|\xi|^{|\nu|} D^{\nu}\left(|\xi|^{-\alpha} \hat{f}(\xi)\right)$ is bounded on $\mathbf{R}^{n}$ for all $\nu$ such that $0 \leq|\nu| \leq m$. Then $f$ is continuous and $f(x)=O\left(|x|^{-n-\alpha}\right)$ as $|x| \rightarrow \infty$.

ProOF. Since $\hat{f}$ is in $L^{1}\left(\mathbf{R}^{n}\right), f$ is continuous and bounded and it suffices to show that

$$
|f(x)| \leq C|x|^{-n-\alpha} \quad \text { if }|x|>1 .
$$

To see (2.8) let $|x|>1$, set $r=|x|, x^{\prime}=x / r$, and write

$$
\begin{aligned}
f(x) & =(2 \pi)^{-n / 2} \int|\xi|^{\alpha} h(\xi) e^{i\langle x, \xi\rangle} d \xi \\
& =(2 \pi)^{-n / 2} r^{-n-\alpha} \int|\xi|^{\alpha} h\left(\frac{\xi}{r}\right) e^{i\left\langle x^{\prime}, \xi\right\rangle} d \xi,
\end{aligned}
$$

where $h(\xi)=|\xi|^{-\alpha} \hat{f}(\xi)$.

The last formula indicates that to verify (2.8) it suffices to show that the Fourier transform of $h_{r}(\xi)=|\xi|^{\alpha} h(\xi / r)$ is bounded on the unit sphere independent of $r>1$.

To see this let $\varphi$ be a function in $C^{\infty}\left(\mathbf{R}^{n}\right)$ such that $\varphi(\xi)=1$ for $|\xi|<1$ and $\varphi(\xi)=0$ for $|\xi|>2$ and write $h_{r}=h_{0}+h_{\infty}$ where $h_{0}(\xi)=h_{r}(\xi) \varphi(\xi)$ and $h_{\infty}(\xi)=h_{r}(\xi)(1-\varphi(\xi))$. Since $\left\|h_{0}\right\|_{1}$ is bounded independent of $r$ it follows that $\hat{h}_{0}(x)$ is bounded for all $x$ and hence is certainly bounded on the unit sphere. To obtain a similar conclusion concerning $h_{\infty}$ write

$$
D^{\nu} h_{\infty}(\xi)=A(\xi)+B(\xi)
$$


where

$$
A(\xi)=\sum c_{\mu}|\xi|^{\alpha-|\mu|} D^{\nu-\mu} h(\xi / r) r^{-|\nu-\mu|}(1-\varphi(\xi)),
$$

the $c_{\mu}$ 's are appropriate constants, and the sum is taken over all multi-indices $\mu$ such that $0 \leq \mu \leq \nu . B(\xi)$ is a similar expression except that each term contains a derivative of $(1-\varphi)$ of order one or higher. Observe that

$$
|\xi|^{\alpha-|\mu|} D^{\nu-\mu} h(\xi / r) r^{-|\nu-\mu|}=|\xi|^{\alpha-|\nu|}\left(|\xi / r|^{|\nu|-|\mu|} D^{\nu-\mu} h(\xi / r)\right)
$$

and the expression in parentheses is bounded by virtue of statement (ii) in the hypothesis. Thus

$$
|A(\xi)| \leq C|\xi|^{\alpha-|\nu|}(1-\varphi(\xi)) .
$$

A similar observation shows that $B$ is supported in $1 \leq|\xi| \leq 2$ and is bounded. These facts concerning $A$ and $B$ imply that

$$
\left|D^{\nu} h_{\infty}(\xi)\right| \leq c|\xi|^{\alpha-|\nu|}(1-\varphi(\xi)) .
$$

Now, if $|\nu|=m$, recalling that $m$ is greater than $n+\alpha$, it follows from (2.11) that $\left\|D^{\nu} h_{\infty}\right\|_{1}$ is bounded independent of $r$. Since this is true for all such $\nu$, we may conclude that $\left.|| x\right|^{m} \hat{h}_{\infty}(x) \mid$ is bounded independent of $r$ and hence $\hat{h}_{\infty}$ is bounded on the unit sphere. This implies the desired result.

3. The range theorem and corollaries. It is convenient to introduce the following definition.

DEFINITION. A function $g$ on $S^{n-1} \times \mathbf{R}$ is said to be uniformly integrable if there exists an integrable function $h$ on $\mathbf{R}$ such that $|g(\theta, t)| \leq h(t)$ for all $\theta$ and $t$.

The range theorem follows.

THEOREM. Let $g \in C^{r}\left(S^{n-1} \times \mathbf{R}\right)$, where $r \geq m=n+2+k, k \geq-1$. Assume $g(-\theta,-t)=g(\theta, t)$,

$$
\begin{aligned}
& t^{k+1+q} D_{\theta}^{j} g^{(q)}(\theta, t) \text { is uniformly integrable for } j=0,1, \ldots, m-q, \\
& \text { and } q=0,1, \ldots, n+1 ; \text { and } \\
& \int_{-\infty}^{\infty} g(\theta, t) t^{l} d t=P_{l}(\theta) \text { is representable as a homogeneous polyno- } \\
& \text { mial of degree } l \text { for } l=0,1, \ldots, k \text {. }
\end{aligned}
$$

Then there exists a function $f$ in $C^{s}\left(R^{n}\right)(s=r+1-n, n$ odd and $s=r-n, n$ even), such that $f(x)=O\left(|x|^{-n-k-1}\right)$ as $|x| \rightarrow \infty$ and $R f(\theta, t)=g(\theta, t)$ for all $\theta$ and $t$. Moreover, for each $x \in \mathbf{R}^{n}$ the inversion formula

$$
f(x)=2^{-1}(2 \pi)^{1-n} \Lambda^{n-1} R^{t} g(x)
$$

holds.

REMARK. When $k=-1,(3.2)$ is taken to be vacuous.

PROOF. The continuity of $g$ and (3.1) with $q=j=0$ imply that $g$ is uniformly integrable. Thus, the function $\hat{f}$ defined in polar coordinates on $\mathbf{R}^{n}$ by

$$
\hat{f}(\tau \theta)=(2 \pi)^{(1-n) / 2} \hat{g}_{\theta}(\tau)=(2 \pi)^{-n / 2} \int_{-\infty}^{\infty} g(\theta, t) e^{-i t \tau} d t
$$


is bounded on $\mathbf{R}^{n}$ and continuous on $\mathbf{R}^{n}-\{0\}$. We show that $\hat{f}$ satisfies the hypotheses of the Lemma with $\alpha=k+1$. Leibnitz formula gives

$$
\left(\frac{d}{d t}\right)^{n+1}\left(t^{m} g\right)=\sum_{q=0}^{n+1} c_{m, k} t^{k+1+q} g^{(q)}
$$

with the $c_{m, k}$ known constants. By (3.1) each term on the right side of (3.5) is uniformly integrable and hence so is the left side. Since $g^{(n+1)}$ is continuous, solving for $g^{(n+1)}$ in (3.5) shows that $g^{(n+1)}$ is uniformly integrable. Thus $\hat{g}$ is continuous on $S^{n-1} \times \mathbf{R}$ and uniformly $O\left(\tau^{-n-1}\right)$ as $\tau \rightarrow \infty$. This, (3.4), and the fact that $\hat{f}$ is bounded imply that $\hat{f}$ is integrable and hence its inverse Fourier transform $f$ is continuous.

We will show at the end of the proof that the polynomial condition (3.2) can be replaced by

$$
\int_{-\infty}^{\infty} g(\theta, t) t^{l} d t=0, \quad l=0,1, \ldots, k,
$$

without loss of generality. Accepting this for now, the uniform integrability assumption of (3.1) allows differentiation under the integral sign in (3.6) giving

$$
\int_{-\infty}^{\infty} D_{\theta}^{j} g(\theta, t) t^{l} d t=0, \quad l=0,1, \ldots, k ; j=0,1, \ldots, m .
$$

Since $t^{k+1} D_{\theta}^{j} g$ is uniformly integrable, the above and Taylor's theorem give that

$$
\left|\left(t^{p} D_{\theta}^{j} g\right)^{\curlyvee}(\tau)\right|=O\left(\tau^{k+1-p}\right) \quad \text { as } \tau \rightarrow 0, \text { when } 0 \leq p \leq k+1 .
$$

Let $0 \leq|\nu| \leq m=n+2+k$. By (3.4), in polar coordinates $|\xi|^{|\nu|} D^{\nu}\left(|\xi|^{-k-1} \hat{f}\right)$ is a sum of terms of the form $\tau^{l}(\partial / \partial \tau)^{l}\left(\tau^{-k-1}\left(D_{\theta}^{j} g\right)^{\wedge}(\tau \theta)\right), l=0,1, \ldots,|\nu| \leq m$, $j=0,1, \ldots,|\nu|-l$. This, in turn, is a sum of terms of the form

$$
\tau^{-k-1+p}\left(t^{p} D_{\theta}^{j} g\right)^{-}(\tau \theta), \quad 0 \leq p \leq l \leq|\nu| \leq m,
$$

where the Fourier transform is in the sense of distributions when $p>k+1$. If $0 \leq p \leq k+1,(3.7)$ and the uniform integrability assumption (3.1) show that terms of the form (3.8) are continuous on $\mathbf{R}^{n}-\{0\}$ and bounded independent of $\theta$. Suppose $m \geq|\nu| \geq l \geq p \geq k+1$. Leibnitz formula shows that $(d / d t)^{p-k-1}\left(t^{p} D_{\theta}^{j} g\right)$ is a sum of terms of the form $t^{k+1+q} D_{\theta}^{j} g^{(q)}, q=0,1, \ldots, p-k-1$. Each such term is uniformly integrable by (3.1). Thus $\left(t^{p} D_{\theta}^{j} g\right)^{\wedge}(\tau \theta)$ is continuous on $\tau>0$ and uniformly $O\left(\tau^{k+1-p}\right)$ as $\tau \rightarrow \infty$ and 0 . Hence (3.8) is bounded in this case also. The Lemma now implies that $f(x)=O\left(|x|^{-n-k-1}\right)$ as $|x| \rightarrow \infty$.

Since $f$ is continuous and $O\left(|x|^{-n}\right)$ as $|x| \rightarrow \infty, R f$ is continuous also. Moreover $f \in L^{p}\left(\mathbf{R}^{n}\right)$ for all $p>1$. By Remark (2.7), (2.2) holds a.e. This, together with (3.4), shows that $R f=g$ a.e. and thus, by continuity, everywhere. Again Remark (2.7) gives that the inversion formula (2.6), and hence (3.3), holds a.e. To show that it holds everywhere it suffices to show that the right hand side of (3.3) is continuous. If $n$ is odd, then (2.3), (2.5) and the fact that $g \in C^{r}\left(S^{n-1} \times \mathbf{R}\right)$ show that the right hand side of (3.3), and hence $f$, is $C^{r+1-n}$. If $n$ is even, then write $\Lambda^{n-1}=\Lambda\left(\Delta^{(n-2) / 2}\right)$ and use the Calderón-Zygmund representation of $\Lambda,(2.4)$. This, together with the fact that the Riesz transforms, $\mathscr{H}_{j}$, reduce differentiability 
by at most $\varepsilon$ for any $\varepsilon>0$, shows that the right side of (3.3), and hence $f$, is $C^{r-n}$. This completes the proof of the Theorem with the exception of showing that, without loss of generality, (3.2) can be replaced by (3.6). To see this, note that by [10, Lemma 7.4], there exists a $C^{\infty}$ function $h$ vanishing outside of the unit ball in $\mathbf{R}^{n}$ such that

$$
\int_{-\infty}^{\infty} R_{\theta} h(t) t^{l} d t=P_{l}(\theta), \quad l=0,1, \ldots, k
$$

where the $P_{l}$ are the same polynomials as those that appear in (3.2). Replacing $g$ by $g-R h$ does not effect (3.1) but replaces (3.2) by (3.6), and $f$ by $f-h$ which has no effect on the smoothness of $f$ or its rate of decay at $\infty$. The proof is complete.

REMARK. The proof actually shows that when $n$ is even then all partial derivatives of $f$ or order $r-n$ satisfy a Hölder condition of order $\alpha$ for any $\alpha<1$. Also, it follows from [9, Theorem 12.6] that the classical Radon inversion formula $f=2^{-1}(2 \pi)^{-n} R^{t} \Lambda^{n-1} g$ is valid everywhere.

We now give a few corollaries of the Theorem.

The Schwartz space $S\left(S^{n-1} \times \mathbf{R}\right)$ consists of those $C^{\infty}$ functions on $S^{n-1} \times \mathbf{R}$ which, together with their derivatives of all orders, decay at infinity faster than the reciprocal of any polynomial in $t$. Then

COROLlaRY $1[\mathbf{1 0}]$. Let $g \in S\left(S^{n-1} \times \mathbf{R}\right)$ and suppose that $g(-\theta,-t)=g(\theta, t)$.

(a) $g=R f$ for some $C^{\infty}$ function $f$ such that $f(x)=O\left(|x|^{-n}\right)$ as $|x| \rightarrow \infty$.

(b) $f(x)=O\left(|x|^{-n-k-1}\right.$ ) if and only if (3.2) holds for $l=0,1, \ldots, k$.

(c) If $f(x)=O\left(|x|^{-m}\right)$ then for all $\nu \in N^{n}, D^{\nu} f(x)=O\left(|x|^{-m-|\nu|}\right)$.

Proof. When $g \in S\left(S^{n-1} \times \mathbf{R}\right),(3.1)$ is satisfied for all choices of $j, q$ and $k$. So (a) and (b) are immediate consequences of the Theorem. To see that (c) holds observe from (2.2) that $R_{\theta} D^{\nu} f=\theta^{\nu}\left(R_{\theta} f\right)^{(|\nu|)}$ and by integration by parts that if $g=R f$ satisfies (3.2) for $l=0,1, \ldots, k$, then $\theta^{\nu} g^{(|\nu|)}$ satisfies (3.2) for $l=0,1, \ldots,|\nu|+k$, and apply (b).

COROLlaRY 2. Let $g \in C^{n+1}\left(S^{n-1} \times \mathbf{R}\right)$ and suppose that $g(-\theta,-t)=g(\theta, t)$. Assume that (3.1) is satisfied with $k=-1$. Then the inversion formula for $R^{t}$,

$$
g=2^{-1}(2 \pi)^{1-n} R \Lambda^{n-1} R^{t} g
$$

holds everywhere.

ProOF. By the Theorem $R f=g$ everywhere with $f$ given by (3.3).

REMARK. Inversion formulas for $R^{t}$ have been given earlier for functions in $S\left(S^{n-1} \times \mathbf{R}\right)$. Helgason [3] gave an inversion formula when (3.6) is satisfied for all nonnegative integers $l$. Gonzalez [2] and Hertle [5] proved that (3.9) holds for $n$ odd and Solmon [10] showed it holds in all dimensions.

The last corollary is a sort of Tauberian theorem.

COROLlaRY 3. Let $g \in C^{n+2}\left(S^{n-1} \times \mathbf{R}\right)$ be such that $g(-\theta,-t)=g(\theta, t)$. Suppose that (3.1) is satisfied for $k=0$. Then $g$ is the Radon transform of an integrable function $f$ if and only if

$$
\int_{-\infty}^{\infty} g(\theta, t) d t=c
$$

where $c$ is a constant, independent of $\theta$. 
PROOF. Since the hypotheses of the Theorem are satisfied with $k=0, g$ is the Radon transform of a continuous function that is $O\left(|x|^{-n-1}\right)$ as $|x| \rightarrow \infty$, and hence of an integrable function. So the condition is sufficient. The necessity of the condition is an immediate consequence of Fubini's theorem.

REMARK. Peters [8] has shown that the Radon transform of the function $f(x)=$ $\sin \left(|x|^{2}\right) /\left(|x|^{2}\right)$, in the plane, is $O\left(|t|^{-2}\right)$ as $t \rightarrow \infty$. Since $f$ is a radial function its Radon transform is independent of $\theta$ and hence so is $\int_{-\infty}^{\infty} R f(\theta, t) d t$. Nevertheless $f$ is not integrable on $\mathbf{R}^{2}$. Hence condition (3.10) is not sufficient by itself to imply the integrability of $f$.

\section{REFERENCES}

1. A. P. Calderón and A. Zygmund, Singular integral operators and differential equations, Amer. J. Math. 79 (1957), 901-921.

2. F. Gonzalez, Radon transforms on Grassmann manifolds, Ph.D. Thesis, M.I.T., Cambridge, Mass., 1984.

3. S. Helgason, The Radon transform on Euclidean spaces, compact two point homogeneous spaces and Grassmann manifolds, Acta Math. 113 (1965), 153-180.

4. _ The Radon transform, Birkhäuser, Boston, Mass., 1980.

5. A. Hertle, Habilitationsschrift, Univ. Mainz (to appear).

6. P. D. Lax and R. S. Phillips, The Paley-Wiener theorem for the Radon transform, Comm. Pure Appl. Math. 23 (1970), 409-424.

7. W. R. Madych, On Littlewood-Paley functions, Studia Math. 50 (1974), 43-63.

8. J. Peters, A Tauberian theorem for the Radon transform, Houston J. Math. 8 (1982), 565-574.

9. K. T. Smith, D. C. Solmon and S. L. Wagner, Practical and mathematical aspects of the problem of reconstructing objects from radiographs, Bull. Amer. Math. Soc. 83 (1977), 1227-1270.

10. D. C. Solmon, Asymptotic formulas for the dual Radon transform and applications, Math. Z. 195 (1987), 321-343.

Department of Mathematics, University of Connecticut, Storrs, ConnecTICUT 06268

Department of Mathematics, Oregon State University, Corvallis, Oregon 97331 